\title{
Wassertemperaturen in einem nordnorwegischen küstennahen Fluß
}

Reinhard Mook

Der gebirgige Inselgürtel Nordnorwegens ist reich an kleinen Flüssen mit einer durchschnittlichen Abflußintensität von $10^{4}$ bis $10^{5} \mathrm{~m}^{3} \mathrm{~d}-1$ aus Niederschlagsgebieten der Größenordnung $10 \mathrm{~km}^{2}$. Im folgenden wird vom Verhalten der Wassertemperatur in einem solchen Fluß berichtet.

\section{Flußbeschreibung}

Beim untersuchten Fluß handelt es sich um Storelva auf der Insel Kvaløya. Er mündet in den Sandnessund. Das horizontalprojizierte Niederschlagsgebiet ist $6 \mathrm{~km}^{2}$ groß und umfaßt einen Höhenbereich vom Meeresspiegel bis $770 \mathrm{~m}$. Es kann folgendermaßen (vgl. Mook, 1965) beschrieben werden:

\begin{tabular}{|c|c|c|c|c|}
\hline 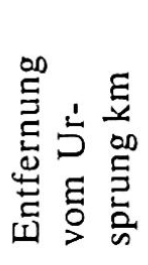 & 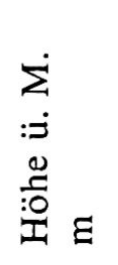 & 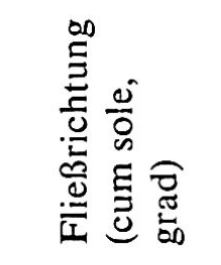 & Kanalform & Gelände \\
\hline 0 & 720 & 220 & Gerinne, Bach & $\begin{array}{l}\text { Schild ohne ausgeprägte Tal- } \\
\text { form. Tundra auf Fels }\end{array}$ \\
\hline 1,7 & 490 & 170 & $\begin{array}{l}\text { Uber Fels schießendes Wasser- } \\
\text { band }\end{array}$ & $\begin{array}{l}\text { Talabschließender Hang, } \\
\text { Tundra }\end{array}$ \\
\hline 2,5 & 300 & 110 bis 200 & $\begin{array}{l}\text { Fluß mit Schnellen. Bäche von } \\
\text { Talseiten }\end{array}$ & $\begin{array}{l}\text { Schuttgefülltes U-Tal, bis } 0,7 \mathrm{~km} \\
\text { breiter Boden, relative Hang- } \\
\text { höhe } 300 \mathrm{~m} \text {. Heidevegetation }\end{array}$ \\
\hline 5,5 & 170 & 170 & $\begin{array}{l}\text { Wasserfälle, 5-10 m tiefer } \\
\text { Geländeeinschnitt }\end{array}$ & $\begin{array}{l}\text { Abfall zur Strandebene. Heide } \\
\text { mit Birkenwald }\end{array}$ \\
\hline 6,3 & 60 & 150 & $\begin{array}{l}\text { Fluß mit Schnellen. Kaum Ober- } \\
\text { flächenzufluß }\end{array}$ & $\begin{array}{l}\text { Strandebene. Moor, Tümpel, } \\
\text { Felsinseln. Birkenwald. Kulti- } \\
\text { viertes Land an Küste }\end{array}$ \\
\hline 7,7 & 0 & & Mündung & \\
\hline
\end{tabular}

Das Wasser wird auf seinem Weg ständig intensiv durchmischt. Bei südlicher Richtungskomponente liegt das Flußbett für kurzwellige Einstrahlung am Mittag weitgehend frei. Die Flußtiefe beträgt gewöhnlich nur einige Dezimeter, so daß ein wesentlicher Teil der durchdringenden Strahlung am Flußboden absorbiert werden dürfte. Im Winter bildet sich über dem Fluß eine bis zu mehreren Metern mächtige Schneedecke aus. Zu Eisbildung kommt es nicht. Die Schneedecke bleibt bis April oder Mai bestehen, d. h. bis zum Zeitpunkt, an dem die Tauflut einsetzt. Diese setzt sich mit Unterbrechungen bis in den Juli fort. Von September oder Oktober an sind starke Niederschläge (Niederschlagsmaximum im Herbst) mit zeitweiser Akkumulation und Taufluten häufig, bis Dezember oder Januar. Die ent- sprechenden jahreszeitlichen Änderungen der Abflußintensität gehen aus Tabelle 1 hervor, die aus dreijährigen Limnigrammen von einem Punkt nahe der Flußmündung abgeleitet wurde. Die Monate sind nach dem hydrologischen Jahr aufgeführt.

Dem Abflußregime nach läßt sich Storelva als Küstenfluß mit kontinentaler Überprägung in den von Mook (1967) angestellten regionalen Vergleich einordnen.

\section{Wassertemperatur}

Die Gleichung des Wärmeumsatzes an einer massenlosen Fläche lautet:

$\mathrm{H}+\mathrm{A}+\mathrm{B}+\mathrm{S}+\mathrm{L}+\mathrm{F}=\mathrm{O}$ 
Tabelle 1

Kennzeichen für den Jahresgang der Abflußintensität $\left(10^{3} \mathrm{~m}^{3} \mathrm{~d}-1\right)$ in Storelva.

Periode 1963 bis 1965 mit 1096 Tageswerten

$10^{3} \mathrm{~m}^{3} \mathrm{~d}-1$ Sept. Okt. Nov. Dez. Jan. Febr. März April Mai Juni Juli Aug. Jahr

$\begin{array}{lrrrrrrrrrrrrr}\text { Minimum } & 4 & 6 & 6 & 3 & 2 & 2 & 1 & 1 & 11 & 42 & 41 & 7 & 1 \\ 1 . \text { Viertel } & 23 & 26 & 22 & 8 & 4 & 4 & 3 & 5 & 24 & 95 & 63 & 23 & 14 \\ \text { Mittel } & 43 & 43 & 36 & 25 & 15 & 13 & 12 & 13 & 77 & 120 & 92 & 36 & 36 \\ \text { 3. Viertel } & 64 & 76 & 74 & 61 & 110 & 22 & 21 & 23 & 150 & 170 & 110 & 41 & 79 \\ \text { Maximum } & 200 & 480 & 320 & 160 & 300 & 115 & 75 & 340 & 260 & 320 & 280 & 75 & 480\end{array}$

Hier stehen $\mathrm{H}$ für den Wärmeaustausch mit dem örtlichen Wasser, A für jenen mit dem advektiv herangeführten Wasser (einschließlich Niederschlag), B für jenen mit dem Flußbett, $S$ für den Strahlungsaustausch und $\mathrm{L}$ und $\mathrm{F}$ für den Übergang sensibler Wärme (Luft) und latenter Wärme (Feuchte). Die Wassertemperatur ist an einem festen Meßpunkt durch den Wärmeaustausch mit der Wassermenge in der Zeiteinheit bestimmt, integriert über Flußlauf und Fließzeit.

Im Winter, wenn Fluß und Boden durch die Schneedecke abgeschirmt sind und keine Schmelze eintritt, halten sich die Variablen der Wärmeumsatzgleichung quasi-konstant oder nehmen vergleichsweise kleine Werte an. Entsprechend hält sich die Wassertemperatur ziemlich konstant. Bei Tauflut kann Wärmezufuhr durch S, L und F je nach Verteilung der schmelzfertigen Schneedecke durch A überkompensiert werden von primärem Schmelzwasser, so daß die Wassertemperatur bei steigendem atmosphärischem Wärmeangebot zunächst sinkt. In jedem Falle wird primäres Schmelzwasser den Temperaturanstieg des Flußwassers dämpfen. Wird die Schneeschmelze durch abnehmendes atmosphärisches Wärmeangebot gedrosselt, kann dies zu steigender Temperatur des Flußwassers führen. - Oberflächenwasser von Regenniederschlag wird mit einer der Luft oder Bodenfläche entsprechenden Temperatur eingehen.

Mit abnehmendem Zufluß von primärem Schmelzwasser wird die Temperatur des Flußwassers an jedem Punkt mehr und mehr von S, L, F und B bestimmt. Diese Variablen kontrollieren dann in steigendem Maße auch die Temperatur des in den Fluß eintretenden Wassers, so daß A abnimmt. Die Temperatur des Flußwassers ist, soweit sie durch unmittelbare Einwirkung der angeführten Variablen bestimmt wird, abhängig von der Abflußintensität. Hierbei handelt es sich um eine Wechselwirkung. Beispielsweise ist $\mathrm{L}+\mathrm{F}$ über den Zustand der Grenzschicht Wasser/Luft, die Strahlungsaufnahme des Bettes über die Transparenz des Wassers abhängig von der Abflußintensität.

Die kurzwellige Strahlungskomponente erhöht, besonders bei direkter Einstrahlung am Sommermit- tag, sowohl über den Boden als das Flußwasser die Flußtemperatur. Kurzwellige Streustrahlung und langwelliger Strahlungsanteil werden in ihrem Einfluß auf die Wassertemperatur meist vom Verlauf anderer Variabler überdeckt. Die Variable L ist durch Strahlung, Feuchteumsatz und Advektion bestimmt. Bei gegebener Luftfeuchte hängt $F$ über den Dampfdruck von der Lufttemperatur ab. Der Wärmeübergang $\mathrm{L}+\mathrm{F}$ ist vom turbulenten Austausch (Wind) abhängig. Unruhige oder gar undefinierte Wasseroberfläche (Wasserfall) fördert den Austausch durch die Grenzschicht Wasser/Luft. Bei hoher Lufttemperatur mit entsprechend hohem Dampfdruck ist mit Kondensation und Wärmeabgabe an kalte Flächen zu rechnen. Bei warmem Flußwasser oder feuchtem Grund tritt Wärmeverlust an die Luft durch Verdunstung ein. Demnach mindert $\mathrm{F}$ bei warmem Wetter die Extreme der Wasser- bzw. Bodentemperatur. Wärmeaustausch mit Wasser, Strahlung und Wärmeleitung bestimmen $B$, dem wie dem übrigen Boden ein langzeitlicher Wärmeausgleich zukommt.

Dominiert eine Variable der Gleichung für den Wärmeumsatz in ihrem Verlauf über die anderen, oder sind diese mit jener funktional verknüpft, so läßt sich die Wassertemperatur in Abhängigkeit von dieser einen Variablen beschreiben. Beispielsweise kann $\mathrm{H}$ als Funktion von $\mathrm{L}$ dargestellt werden, wenn an Sommer- oder Herbstmorgen A und B klein, die übrigen Variablen vernachlässigbar oder genügend über $L$ berücksichtigt sind.

Der Wärmehaushalt fließender Gewässer ist u. a. von Eckel und Reuter (1950) und von Eckel (1953) eingehend behandelt worden. Hier nun sollen keine quantitativen Abschätzungen versucht, sondern ausgehend von den vorstehenden Überlegungen geographisch relevante Eigenschaften im Verhalten der Wassertemperatur an einer festen Meßstelle beschrieben werden. Um Hinweise auf die thermischen Verhältnisse in Flüssen der Art von Storelva zu erhalten, wurde im Auftrag des norwegischen Amtes für Wasser- und Elektrizitätswirtschaft während der Jahre 1963 bis 1965 zweimal täglich, um 6 und 12 Uhr, die Wassertemperatur gemessen. Verwendet wurde ein $\mathrm{Hg}$-Thermometer mit Präzisions- 
Tabelle 2

Kennzeichen für den Jahresgang der Wassertemperatur $\left({ }^{\circ} \mathrm{C}\right)$ um 6 und 12 Uhr in Storelva

Periode 1963 bis 1965 mit 1096 Tagen

Minimum

${ }^{\circ} \mathrm{C}$

Sept. Okt. Nov. Dez. Jan. Febr. März April Mai Juni Juli Aug. Jahr

$6 \mathrm{Uhr}$

$\begin{array}{llll}1,0 & 0,15 & 0,05 & 0,05\end{array}$

$0,10 \quad 0,05$

$\begin{array}{llll}0,05 & 0,05 & 0,05 & 0,10\end{array}$

$2,2 \quad 5,5 \quad 0,05$

$12 \mathrm{Uhr}$

$\begin{array}{llll}3,4 & 0,15 & 0,05 & 0,05\end{array}$

$0,10 \quad 0,10$

$\begin{array}{llll}0,10 & 0,05 & 0,05 & 0,40\end{array}$

$2,9 \quad 7,2 \quad 0,05$

1. Viertel

$6 \mathrm{Uhr}$

$\begin{array}{lll}4,7 & 0,80 & 0,33\end{array}$

$\begin{array}{lll}0,25 & 0,18 & 0,09\end{array}$

$\begin{array}{llll}0,08 & 0,16 & 0,42 & 1,70\end{array}$

$\begin{array}{lll}5,1 & 7,1 & 0,23\end{array}$

$12 \mathrm{Uhr}$

$6,4 \quad 1,20 \quad 0,34 \quad 0,22$

$0,20 \quad 0,10$

$\begin{array}{llll}0,10 & 0,12 & 0,45 & 2,30\end{array}$

$7,8 \quad 8,2 \quad 0,25$

Mittel

$6 \mathrm{Uhr}$

$5,9 \quad 2,30 \quad 0,4$

$0,40 \quad 0,34$

$0,22 \quad 0,20$

$\begin{array}{llll}0,15 & 0,20 & 0,75 & 2,80\end{array}$

$\begin{array}{rrr}6,8 & 8,7 & 0,43\end{array}$

$12 \mathrm{Uhr}$

$\begin{array}{llll}7,8 & 4,10 & 0,42 & 0,26\end{array}$

$0,24 \quad 0,18$

$\begin{array}{llll}0,15 & 0,18 & 1,00 & 3,90\end{array}$

$10,9 \quad 11,3 \quad 0,45$

3. Viertel

$6 \mathrm{Uhr}$

$\begin{array}{lll}7,2 & 2,80 & 0,45\end{array}$

$\begin{array}{lll}0,40 & 0,37 & 0,35\end{array}$

$\begin{array}{llll}0,28 & 0,35 & 1,30 & 4,00\end{array}$

$8,1 \quad 10,7 \quad 4,60$

$12 \mathrm{Uhr}$

$9,8 \quad 5,00$

0,47

$0,30 \quad 0,30 \quad 0,25$

$\begin{array}{llll}0,25 & 0,28 & 1,90 & 5,70\end{array}$

$12,5 \quad 12,8 \quad 7,20$

Maximum

$6 \mathrm{Uhr}$

$10,6 \quad 5,50$

$4,50 \quad 0,50 \quad 0,55$

0,50

$0,45 \quad 0,50$

$3,20 \quad 8,30$

$11,3 \quad 13,5 \quad 13,50$

$12 \mathrm{Uhr}$

$$
12,0 \quad 7,40
$$

$4,70 \quad 0,35$

$0,35 \quad 0,50$

$\begin{array}{lllllll}0,50 & 0,50 & 4,70 & 12,30 & 15,7 & 17,0 & 17,00\end{array}$

skala und üblichem Wasserkörper. Die Meßstelle lag wenig oberhalb der Flußmündung. Durch die rege Mischung des Wassers war ein quasi-homogenes Temperaturfeld quer zur Strömungsrichtung gegeben. Die Verteilung der meteorologischen Variablen während des Beobachtungszeitraumes paßten cum grano salis in das langjährige Muster.

Wie Tabelle 2 zeigt, wurden die tiefsten Wassertemperaturen von Oktober bis Juni, d. h. zur Zeit der Taufluten und während des dazwischenliegenden Winters gemessen. Mit fortschreitender Ausaperung, abnehmendem Einfluß primären Schmelz- wassers und mit der Erwärmung des Bodens stieg die Wassertemperatur im Juli kräftig an und kulminierte im August (einen Monat nach der Lufttemperatur). Mit zunehmender Abkühlung (Ausstrahlungsüberschuß) und zeitweiser Akkumulation von Schnee sank die Wassertemperatur im September und Oktober rasch ab. - Bei über der Hälfte aller Beobachtungen lag die Wassertemperatur unter $0,5^{\circ} \mathrm{C}$.

Vom November an fällt die kurzwellige Strahlung, welche eine regelmäßige Tagesperiode des Wärmehaushaltes verursacht, fort. Bis April schirmte eine

\section{Tabelle 3}

Jahresgang der Amplitude der Wassertemperatur 12 minus 6 Uhr in Storelva, 1963 bis 1965. Häufigkeit in Promille der 1096 Wertepaare

Amplitude

$0^{\circ} \mathrm{C}$

$-<1.01$

$-0,51-1,00$

Sept. Okt. Nov. Dez. Jan. Febr. März April Mai Juni Juli Aug. Jahr

$-0,01-0,50$

12

$\overline{+0,00-0,49} \quad 2 \quad 29$

$+0,50-0,99 \quad 14 \quad 17$

$+1,00-1,99 \quad 39 \quad 23$

$+2,00-2,99 \quad 17 \quad 3$

$+3,00-3,996$

$+4,00-5,992$

$+6,00-7,99$

$+\geq 8.00$

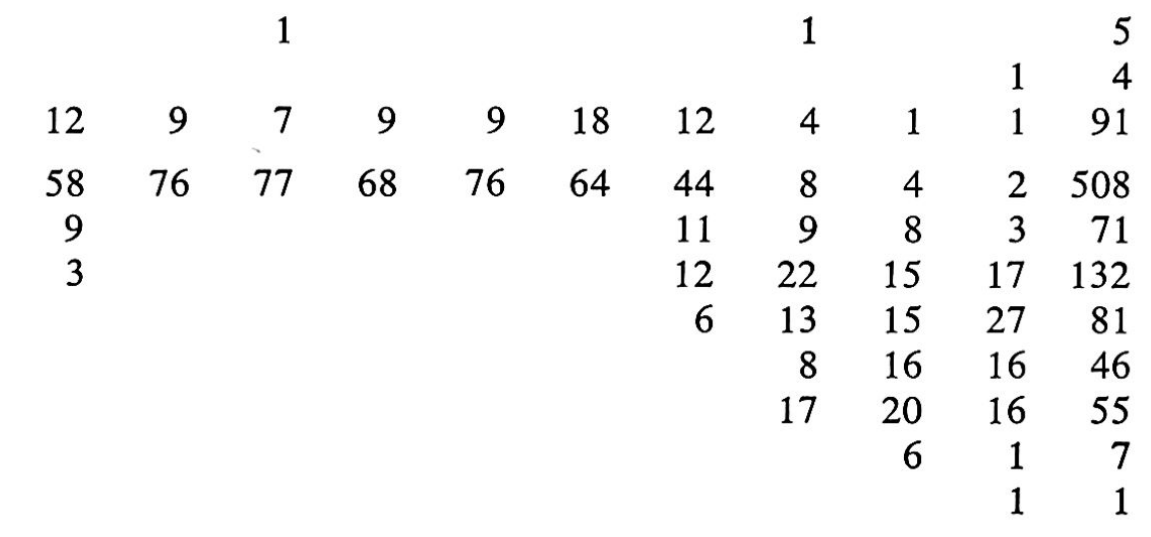


Schneedecke Niederschlagsfeld und Fluß. Die Amplitude der Wassertemperatur 12 und 6 Uhr war in dieser Zeit nahezu null. Möglicherweise war das Flußwasser dem thermischen Gleichgewicht mit seiner Umgebung nahe. Während der Tauflut dämpfte primäres Schmelzwasser die Amplitude der Wassertemperatur. Dies geht auch aus Tab. 3 über die Häufigkeiten der Amplitude hervor. Große Schwankungen (bis etwa $7^{\circ}$ ) traten erst im Juli auf. Im Mittel erreichten die Amplituden im Juli/August $3^{\circ} \mathrm{C}$. Negative Schwankungen, d.h. Mittagstemperatur niedriger als Morgentemperatur, kamen vor allem im Herbst und Frühjahr, bedingt durch Taufluten, vor. Im übrigen spiegeln die negativen Schwankungen den Einfluß von Wetterveränderungen am Vormittag.

An einigen Beispielen soll das Verhalten der Wassertemperatur noch näher erläutert werden. Als verfügbare Indikatoren für das atmosphärische Wärmeangebot werden die Extreme der Lufttemperatur $\mathrm{T}_{1}$, der Bewölkungsgrad in Oktas $(\mathrm{N})$ und die Niederschlagsmenge $\mathrm{P}$ in $\mathrm{mm}$ angezogen. Diese Daten gelten für das benachbarte Tromsö. Die Wassertemperatur wird mit $\mathrm{Tw}$ symbolisiert. Andere als interdiurne Änderungen bleiben außer Betracht.

In Tabelle 4 sind Mittelwerte für Tw 6 und $12 \mathrm{Uhr}$ im April nach $T_{1}$ geodnet. Das noch ganz mit Schnee bedeckte Niederschlagsfeld verursachte durch Schmelzwasser über die Größe A der Wärmeumsatzgleichung bei steigendem positivem $T_{1}$ fallendes Tw.

\section{Tabelle 4}

Storelva, April 1963 bis 1965. Mittelwerte für die Wassertemperatur $T w$ in Abhängigkeit von den Extremen der Lufttemperatur $\mathrm{T}_{1} .\left({ }^{\circ} \mathrm{C}\right) \mathrm{Zahl}$ der Fälle $n$.

$\begin{array}{lllllll}\text { Minimum } \mathrm{T}_{1} & -10,6 & -7,2 & -2,8 & 0,8 & 2,9 & 5,1 \\ \text { Tw 6 Uhr } & 0,18 & 0,25 & 0,27 & 0,12 & 0,08 & 0,05 \\ \text { n } & 1 & 23 & 34 & 16 & 13 & 3 \\ \text { Maximum } T_{1} & & -2,8 & 1,1 & 3,2 & 6,6 \\ \text { Tw 12 Uhr } & & 0,25 & 0,15 & 0,10 & 0,05 \\ \text { n } & & 15 & 22 & 16 & 37\end{array}$

Am Beispiel fast willkürlich herausgegriffener Junitage 1965 sei die Depression von Tw um 12 Uhr durch Schmelzwasser gezeigt (Tabelle 5): Vom 5. zum 6. steigendes $T_{1}$ (Wärmeangebot) förderte die Schmelze, so daß Tw bei unveränderter Einstrahlung (Bewölkungsgrad $\mathrm{N}$ ) nahezu unverändert blieb. Kräftige Einstrahlung am 7. ergab durch primäres Schmelzwasser nur gedämpft steigendes Tw. Bei etwas geringerer Einstrahlung am 8. sank $T w$ bei konstantem $T_{1}$. Im weiteren Verlauf stieg $\mathrm{Tw}$ bei fallendem $\mathrm{T}_{1}$ und bewölktem Himmel, da nun der Zufluß primären Schmelzwassers gedrosselt war.

\section{Tabelle 5}

Storelva. Beispiel für den Gang der Wassertemperatur an Junitagen 1965. Symbole siehe Text

Datum: Juni 1965

$\begin{array}{lccccccl} & 5 . & 6 . & 7 . & 8 . & 9 . & 10 . & 11 . \\ \mathrm{T}_{1} \max & 7,8 & 12,5 & 16,8 & 16,8 & 14,6 & 8,2 & 5,1 \\ \mathrm{Tw} 12 \mathrm{Uhr} & 0,4 & 0,5 & 1,4 & 1,0 & 1,2 & 2,0 & 2,0 \\ \mathrm{~N} & 8 & 7 & 0 & 3 & 7 & 4 & 8 \\ \mathrm{P} & 1 & & & & 1 & & \end{array}$

Wie Mook (1965) ausführte, kann die Schmelzwassermenge bei bekannter Verteilung der Schneedecke im Gelände aus meteorologischen Daten abgeschätzt werden. Unstetigkeiten im zeitlichen Verlauf von Temperatur und Wasserführung an einer Meßstelle zeigen das Eintreffen des für einen bestimmten Bereich errechneten Schmelzwassers an. Auf diese Weise konnte für Storelva eine mittlere fiktive Rinnund Fließgeschwindigkeit vom Schwerpunkt des Schmelzbereiches bis nahe der Mündung zu $1 \mathrm{~m}$ sec-1 abgeschätzt werden. Dieser Wert dürfte der Größenordnung nach auch außerhalb der Tauperiode gültig sein. Die mittlere zeitliche Weglänge des Flußwassers, als Randbedingung für die beobachteten Temperaturen, würde dann etwa 1 Stunde oder wenig mehr betragen.

Im Sommer macht sich während meteorologisch kühler Abschnitte die Wärmekapazität des Bodens, des gespeicherten Wassers und des Bettes auf den Gang der Wassertemperatur ausgleichend bemerkbar. Niederschlag wirkt sich vergleichsweise kurzzeitig aus. Tabelle 6 zeigt am Beispiel von Anfang August 1963, daß Rückgang von $T_{1}$ und kurzwelliger Strahlung (zunehmendes $\mathrm{N}$ ) den Wert Tw kaum ändert. Am niederschlagreichen 3. entsprach $T w$ dem durchschnittlichen $T_{1}$. Bei sinkendem $T_{1}$ am 4. nahm $T w$ trotz fehlender direkter Einstrahlung nur wenig ab. Diese ausgleichende Wirkung gespeicherter Wärme ergab mit Einstrahlung am 5. nach Abfluß des Niederschlagsüberschusses kräftig ansteigendes Tw, das dann am 6. über dem Ausgangswert vom 1. lag.

\section{Tabelle 6}

Storelva. Beispiel für den Gang der Wassertemperatur an Augusttagen 1963. Symbole siehe Text

Datum August

$\begin{array}{lcccccc} & 1 . & 2 . & 3 . & 4 . & 5 . & 6 . \\ \mathrm{T}_{1} \max & 20,3 & 17,3 & 13,1 & 9,3 & 13,2 & 18,2 \\ \mathrm{Tw} 12 \mathrm{Uhr} & 15,6 & 15,5 & 12,5 & 10,7 & 15,2 & 16,5 \\ \mathrm{~N} & 1 & 5 & 8 & 8 & 0 & 2 \\ \mathrm{P} & & & 20 & & & \end{array}$

In Perioden ohne Schneeschmelze oder Niederschlag folgt bei tiefstehender oder fehlender Sonne die Wassertemperatur im statistischen Mittel der 
Lufttemperatur. Dies bedeutet, daß das Verhalten von $\mathrm{H}$ durch die Variable L der Wärmeumsatzgleichung beschrieben werden kann. Für entsprechende Tage sind die Werte Tw von $6 \mathrm{Uhr}$ und $\mathrm{T}_{1}$ min aus der Zeit von Juli bis Oktober in Tabelle 7 aufgenommen. Der statistische Zusammenhang zwischen den beiden Variablen erwies sich als annähernd linear. Bei niederem $T_{1}$ war $T w>T_{1}$, d. h. das Wasser wärmer als die Luft. Bei hohem $T_{1}$ war $T w \leqslant T_{1}$, d. h. etwas kühler oder von gleichem Betrag wie die Luft. Im wesentlichen war demnach die Wassertemperatur nicht nur im Verlauf, sondern auch dem Betrag des in Tromsö gemessenen Minimums der Lufttemperatur angeglichen.

\section{Tabelle 7}

Zusammenhang zwischen Wassertemperatur $\mathrm{Tw}$ $6 \mathrm{Uhr}$ in Storelva und Minimum der Lufttemperatur $\mathrm{T}_{1}\left({ }^{\circ} \mathrm{C}\right)$. Ausgewählte Fälle (Anzahl n) Juli bis Oktober 1963/1965

$\begin{array}{ccccccccc}\text { Tw } 6 \text { Uhr } 0,4 & 1,8 & 4,2 & 5,9 & 7,8 & 9,7 & 11,8 & 14,1 \\ \mathrm{~T}_{1} \min & -1,4 & 1,1 & 3,8 & 6,0 & 8,1 & 9,9 & 12,2 & 14,4 \\ \mathrm{n} & 22 & 28 & 46 & 70 & 61 & 48 & 20 & 4\end{array}$

\section{Zusammenfassung}

In einem für die küstennahe Zone Nordnorwegens typischen kleinen Fluß wurde während dreier Jahre zweimal täglich die Wassertemperatur nahe der Mündung gemessen. Während des Winters bis zum April zeigte sich die Wassertemperatur monoton. Dann, bis zum Juli und später wieder im Oktober, dominierte der Einfluß von Taufluten die Temperatur. Die höchsten Wassertemperaturen und Tagesamplituden wurden im August erreicht, wenn Strahlung und Temperatur von Boden und Luft die Wassertemperatur stark prägten.

\section{Summary}

In a small river typical for the sub-coastal belt of Northern Norway, water temperatures near the mouth were measured twice a day through three years. Water temperatures ran monotonously during winter until April. Then, until July, and later in October, runoff from snowmelt governed water temperatures. Highest water temperatures and daily variations in it were observed in August, when insolation and temperature of ground and air strongly influenced on water temperatures.

\section{Literatur}

Eckel, O. und Reuter, H. (1950): Zur Berechnung des sommerlichen Wärmeumsatzes in Flußläufen. Geografiska Annaler. 32, S. 188-209.

Eckel, O. (1953): Zur Thermik der Fließgewässer. Wetter und Leben, Sonderheft 2, S. 41-47.

Mook, R. H. G. (1965): Meteorologische Faktoren im Gelände nahe Tromsö während der Schneeschmelze. Pure and Applied Geophysics, 60, S. 201216.

Mook, R. H. G. (1967): Über Winterklima und Abfluß an der Küste und im Binnenland Nord-Norwegens. Geographica Helvetica, 22, S. 31-35. 